\title{
The ring of modular forms of degree two in characteristic three
}

\author{
Gerard van der Geer ${ }^{1,2}$
}

Received: 28 March 2020 / Accepted: 4 September 2020 / Published online: 16 September 2020

(c) The Author(s) 2020

\section{Abstract}

We determine the structure of the ring of Siegel modular forms of degree 2 in characteristic 3.

Mathematics Subject Classification 11F03 $\cdot 14 \mathrm{~J} 15 \cdot 14 \mathrm{G} 35 \cdot 11 \mathrm{G} 18$

\section{Introduction}

Let $\mathcal{A}_{g}$ be the moduli space of principally polarized abelian varieties of dimension $g$. It is a Deligne-Mumford stack over $\mathbb{Z}$. It carries a natural vector bundle of rank $g$, the Hodge bundle $\mathbb{E}_{g}$. We write $L$ for its determinant line bundle. The vector bundle $\mathbb{E}_{g}$ extends in a natural way over any compactification $\tilde{\mathcal{A}}_{g}$ of Faltings-Chai type and we will denote the extension of $\mathbb{E}_{g}$ and $L$ again by the same symbols. Sections of $L^{\otimes k}$ over $\tilde{\mathcal{A}}_{g}$ are called modular forms of weight $k$. It is known that for $g \geq 2$ any section of $L^{k}$ over $\mathcal{A}_{g}$ extends to a section of $L^{k}$ over $\tilde{\mathcal{A}}_{g}$, a fact usually referred to as the Koecher principle, see [7, Prop. 1.5, p. 140].

If $\mathbb{F}=\mathbb{Z}$ or $\mathbb{Z}_{p}$ or a field one has the graded ring

$$
\mathcal{R}_{g}(\mathbb{F})=\oplus_{k} H^{0}\left(\tilde{\mathcal{A}}_{g} \otimes \mathbb{F}, L^{k}\right) .
$$

It is known by [7] that it is a finitely generated $\mathbb{F}$-algebra.

In the case of $\mathbb{F}=\mathbb{C}$ the ring $\mathcal{R}_{g}(\mathbb{C})$ is the ring of scalar-valued Siegel modular forms of degree $g$. It is well-known known that $\mathcal{R}_{1}(\mathbb{C})=\mathbb{C}\left[E_{4}, E_{6}\right]$ is freely generated over $\mathbb{C}$ by the Eisenstein series $E_{4}$ and $E_{6}$ of weights 4 and 6. In the 1960s Igusa [11] determined the structure of $\mathcal{R}_{2}(\mathbb{C})$ :

$$
\mathcal{R}_{2}(\mathbb{C})=\mathbb{C}\left[\psi_{4}, \psi_{6}, \chi_{10}, \chi_{12}, \chi_{35}\right] /\left(\chi_{35}^{2}-P\right),
$$

where the indices of the generators indicate the weights and $P$ is a polynomial in $\psi_{4}, \psi_{6}, \chi_{10}$ and $\chi_{12}$. Moreover, the ideal of cusp forms is generated by $\chi_{10}, \chi_{12}$ and $\chi_{35}$. For $g=3$,

Gerard van der Geer

g.b.m.vandergeer@uva.nl

1 Korteweg-de Vries Instituut, Universiteit van Amsterdam, Science Park 904, 1098 XH Amsterdam, The Netherlands

2 Université du Luxembourg, Unité de Recherche en Mathématiques, 4364 Esch-sur-Alzette, Luxembourg 
Tsuyumine showed in [20] that $\mathcal{R}_{3}(\mathbb{C})$ is generated by 34 elements; recently the number of generators was reduced to 19 by Lercier and Ritzenthaler [14].

For $\mathbb{F}=\mathbb{F}_{p}$, a finite field with $p$ elements, the ring $R_{1}\left(\mathbb{F}_{p}\right)$ was described by Deligne [5]. Besides giving the structure of the ring over $\mathbb{Z}$

$$
\mathcal{R}_{1}(\mathbb{Z})=\mathbb{Z}\left[c_{4}, c_{6}, \Delta\right] /\left(c_{4}^{3}-c_{6}^{2}-1728 \Delta\right),
$$

he showed that

$$
\mathcal{R}_{1}\left(\mathbb{F}_{2}\right)=\mathbb{F}_{2}\left[a_{1}, \Delta\right] \text { and } \mathcal{R}_{1}\left(\mathbb{F}_{3}\right)=\mathbb{F}_{3}\left[b_{2}, \Delta\right],
$$

where $\Delta$ is of weight 12 and $a_{1}\left(\operatorname{resp} b_{2}\right)$ is of weight 1 (resp. 2). For $p \geq 5$ we have $\mathcal{R}_{1}\left(\mathbb{F}_{p}\right)=\mathbb{F}_{p}\left[c_{4}, c_{6}\right]$.

For $g=2$, Igusa determined in [13] also the ring of modular forms over $\mathbb{Z}$; it is generated by elements of weight

$$
4,6,10,12,12,16,18,24,28,30,35,36,40,42,48 .
$$

For finite fields the structure of $\mathcal{R}_{2}\left(\mathbb{F}_{p}\right)$ is known for $p \geq 5$. For this we refer to Ichikawa's paper [10]. For $p \geq 5$ the ring is just as in characteristic zero generated by modular forms $\psi_{4}, \psi_{6}, \chi_{10}, \chi_{12}$ and $\chi_{35}$ with $\chi_{35}$ satisfying a relation $\chi_{35}^{2}=P\left(\psi_{4}, \psi_{6}, \chi_{10}, \chi_{12}\right)$. Moreover for $p \geq 5$ the reduction map $\mathcal{R}_{2}\left(\mathbb{Z}_{p}\right) \rightarrow \mathcal{R}_{2}\left(\mathbb{F}_{p}\right)$ is surjective. Nagaoka studied the image of the reduction map in [17,18], see also [1].

In this paper we consider the case $p=3$ and determine the structure of $\mathcal{R}_{2}\left(\mathbb{F}_{3}\right)$. We use the close connection between the moduli space $\mathcal{A}_{2}$ and the moduli space $\mathcal{M}_{2}$ of curves of genus 2 via the Torelli map $\mathcal{M}_{2} \hookrightarrow \mathcal{A}_{2}$ and the description of $\mathcal{M}_{2}$ as a quotient stack for the action of GL(2) on the space of binary sextics. In that way invariant theory can be used to construct modular forms. The relation between invariants and modular forms was already exploited by Igusa in [11], but he used theta functions and Thomae's formula to relate these to cross ratios of the zeros of a binary sextic. Here we use not only invariants but also covariants giving vector-valued modular forms as introduced in [2] to analyze the regularity of scalar-valued modular forms.

Our result is:

Theorem 1.1 The subring $\mathcal{R}_{2}^{\mathrm{ev}}\left(\mathbb{F}_{3}\right)$ of modular forms of even weight is generated by forms of weights 2,10,12, 14 and 36 and has the form

$$
\mathcal{R}_{2}^{\mathrm{ev}}\left(\mathbb{F}_{3}\right)=\mathbb{F}_{3}\left[\psi_{2}, \chi_{10}, \psi_{12}, \chi_{14}, \chi_{36}\right] / J
$$

with $J$ the ideal generated by the relation

$$
\psi_{2}^{3} \chi_{36}-\chi_{10}^{3} \psi_{12}-\psi_{2}^{2} \chi_{10} \chi_{14}^{2}+\chi_{14}^{3} .
$$

Moreover, $\mathcal{R}_{2}\left(\mathbb{F}_{3}\right)=\mathcal{R}_{2}^{\mathrm{ev}}\left(\mathbb{F}_{3}\right)\left[\chi_{35}\right] /\left(\chi_{35}^{2}-P\right)$ with $P$ a polynomial in $\psi_{2}, \chi_{10}, \psi_{12}, \chi_{14}$ and $\chi_{36}$. The ideal of cusp forms is generated by $\chi_{10}, \chi_{14}, \chi_{35}, \chi_{36}$.

The generator $\psi_{2}$ is the Hasse invariant that vanishes on the locus of non-ordinary abelian surfaces and $\chi_{10}$ is a form that vanishes on the locus of products of elliptic curves. The ring of modular forms of degree 2 in characteristic 2 is described in [4].

\section{The proof of Theorem 1.1}

Since for $g=2$ the moduli stack $\mathcal{A}_{g} \otimes \mathbb{F}_{3}$ has a canonical compactification due to Igusa we will use this compactification $\tilde{\mathcal{A}}_{2} \otimes \mathbb{F}_{3}$. We will denote the space of sections of $L^{k}$ on 
$\tilde{\mathcal{A}}_{2} \otimes \mathbb{F}_{3}$ by $M_{k}\left(\Gamma_{2}\right)$ and we thus have $\mathcal{R}_{2}\left(\mathbb{F}_{3}\right)=\oplus_{k} M_{k}\left(\Gamma_{2}\right)$. We write $M_{k}\left(\Gamma_{1}\right)$ for the space $H^{0}\left(\tilde{\mathcal{A}}_{1} \otimes \mathbb{F}_{3}, L^{k}\right)$. The Satake compactification is denoted by $\mathcal{A}_{2}^{*} \otimes \mathbb{F}_{3}$. We denote the first Chern class of $L$ by $\lambda_{1}$.

We begin by constructing generators of weight 2 and 10 . The locus $V_{1}$ of abelian surfaces with $p$-rank $\leq 1$ is a divisor in $\mathcal{A}_{2} \otimes \mathbb{F}_{p}$ and its closure $\bar{V}_{1}$ in $\tilde{\mathcal{A}}_{2} \otimes \mathbb{F} p$ has cycle class $(p-1) \lambda_{1}$ in the Chow group with $\mathbb{Q}$-coefficients, so $\left[\bar{V}_{1}\right]=2 \lambda_{1}$ for $p=3$, see $[6,22]$. Therefore the effective divisor $\bar{V}_{1}$ is the divisor of a section of $L^{\otimes 2}$ and there is a modular form $\psi_{2}$ of weight 2 whose zero divisor is $\bar{V}_{1}$. It is determined up to multiplication by a non-zero scalar. We will normalize it later. This form is known as the Hasse invariant. Multiplication by $\psi_{2}$ implies that $\operatorname{dim} M_{k}\left(\Gamma_{2}\right) \leq \operatorname{dim} M_{k+2}\left(\Gamma_{2}\right)$.

The divisor of products of elliptic curves $H_{1}:=\mathcal{A}_{1,1} \otimes \mathbb{F}_{3}$ gives rise to a second modular form. (The notation refers to the fact that $H_{1}$ is the Humbert surface of discriminant 1.) In the Chow group of codimension 1 of $\tilde{\mathcal{A}}_{2} \otimes \mathbb{F}_{3}$ (resp. $\mathcal{A}_{2}^{*} \otimes \mathbb{F}_{3}$ ) we have the relation (cf. e.g. [16, p. 317])

$$
2\left[\bar{H}_{1}\right]+[D]=10 \lambda_{1} \quad\left(\operatorname{resp} . \quad 2\left[\bar{H}_{1}\right]=10 \lambda_{1}\right),
$$

with $D$ the divisor at infinity, hence there exists a modular form of weight 10 vanishing with multiplicity 2 on $H_{1}$. We call this form $\chi_{10}$ (up to a normalization to be determined later). The automorphism group of a generic product of elliptic curves has an extra involution (when compared with the automorphism group of a generic principally polarized abelian surface) and it acts by -1 on $L$, hence every modular form of even weight vanishes with even multiplicity along $H_{1}$.

Restriction to $H_{1}$ yields for even $k$ an exact sequence

$$
0 \rightarrow H^{0}\left(\mathcal{A}_{2} \otimes \mathbb{F}_{3}, L^{k} \otimes O\left(-2 H_{1}\right)\right) \rightarrow H^{0}\left(\mathcal{A}_{2} \otimes \mathbb{F}_{3}, L^{k}\right) \rightarrow H^{0}\left(H_{1}, L_{\mid H_{1}}^{k}\right)
$$

and in view of the degree 2 morphism $\mathcal{A}_{1} \times \mathcal{A}_{1} \rightarrow \mathcal{A}_{1,1}$ induced by interchanging the two factors, we can identify this with

$$
0 \rightarrow M_{k-10}\left(\Gamma_{2}\right) \rightarrow M_{k}\left(\Gamma_{2}\right) \rightarrow \operatorname{Sym}^{2}\left(M_{k}\left(\Gamma_{1}\right)\right),
$$

where the second arrow is multiplication by $\chi_{10}$. Moreover $M_{k-10}\left(\Gamma_{2}\right)=(0)$ for $k<8$ since $L$ is ample on $\mathcal{A}_{2}^{*} \otimes \mathbb{F}_{3}$. The exact sequence (2.1) and the fact that we know $M_{k}\left(\Gamma_{1}\right)$ implies that $\operatorname{dim} M_{k}\left(\Gamma_{2}\right)=1$ for $k=2,4,6,8$ and $\operatorname{dim} M_{10}\left(\Gamma_{2}\right)=2$ and $M_{10}\left(\Gamma_{2}\right)$ is generated by $\psi_{2}^{5}$ and $\chi_{10}$.

We now turn to the construction of the other generators. We use the ideas of [2]. The Torelli map defines an embedding $\mathcal{M}_{2} \otimes \mathbb{F}_{3} \rightarrow \mathcal{A}_{2} \otimes \mathbb{F}_{3}$. A smooth projective curve of genus 2 can be given by an equation

$$
y^{2}=f(x) \quad \text { with } f=\sum_{i=0}^{6} a_{i} x^{6-i} .
$$

We let $V=\left\langle x_{1}, x_{2}\right\rangle$ be the $\mathbb{F}_{3}$-vector space generated by $x_{1}, x_{2}$ and write $f$ as a homogeneous polynomial $\sum_{i=0}^{6} a_{i} x_{1}^{6-i} x_{2}^{i}$. Note that a curve as in (2.2) comes with a basis of the space of regular differentials, viz. $d x / y, x d x / y$.

We have a description of $\mathcal{M}_{2} \otimes \mathbb{F}_{3}$ as the stack quotient $\left[\mathcal{X}^{0} / \mathrm{GL}(V)\right]$ with $\mathcal{X}^{0} \subset \mathcal{X}=$ $\operatorname{Sym}^{6}(V) \otimes \operatorname{det}(V)^{-2}$ the locus given by the non-vanishing of the discriminant, see [3, Section 3, p. 3].

The pullback to $\mathcal{X}^{0}$ of the Hodge bundle under the composition of $\mathcal{X}^{0} \rightarrow \mathcal{M}_{2}$ with the Torelli map $\mathcal{M}_{2} \hookrightarrow \mathcal{A}_{2}$ is the equivariant bundle $V$ on $\mathcal{X}^{0}$ as the basis $d x / y, x d x / y$ of the 
space of regular differentials on the curve $y^{2}=f(x)$ shows. The pullback of $L$ is $\operatorname{det}(V)$. As a consequence pulling back defines a homomorphism

$$
\mu: \mathcal{R}_{2}\left(\mathbb{F}_{3}\right) \rightarrow I
$$

with $I$ the ring of invariants of the action of $\operatorname{GL}(V)$ on $\operatorname{Sym}^{6}(V)$. Here an invariant is a polynomial in $a_{0}, \ldots, a_{6}$, the coefficients of $f$ that is invariant under $\operatorname{SL}(V)$. Since the image of $\mathcal{M}_{2}$ in $\mathcal{A}_{2}$ is a Zariski open part with complement $H_{1}$, not every invariant corresponds to a modular form; but every invariant corresponds to a rational modular form that is regular outside $H_{1}$. In particular, it becomes regular on all of $\mathcal{A}_{2}$ when multiplied with a sufficiently high power of $\chi_{10}$. This provides us with homomorphisms

$$
\mathcal{R}_{2}\left(\mathbb{F}_{3}\right) \stackrel{\mu}{\longrightarrow} I \stackrel{v}{\longrightarrow} \mathcal{R}_{2}\left(\mathbb{F}_{3}\right)_{\chi_{10}},
$$

where $\mathcal{R}_{2}\left(\mathbb{F}_{3}\right)_{\chi_{10}}$ is obtained from $\mathcal{R}_{2}\left(\mathbb{F}_{3}\right)$ by allowing powers of $\chi_{10}$ in the denominator. We have $v \circ \mu=$ id.

This generalizes as follows to vector-valued modular forms. For each finite dimensional irreducible representation $\rho$ of GL(2) there is a vector bundle $\mathbb{E}_{2}^{\rho}$ obtained from $\mathbb{E}_{2}$ by applying a Schur functor. Such a $\rho$ is of the form $\operatorname{Sym}^{j}(\mathrm{St}) \otimes \operatorname{det}^{k}(\mathrm{St})$ with St the standard representation of $\operatorname{GL}(V)$. A section of $\operatorname{Sym}^{j}\left(\mathbb{E}_{2}\right) \otimes \operatorname{det}\left(\mathbb{E}_{2}\right)^{k}$ over $\mathcal{A}_{2}$ is called a modular form of degree 2 and weight $(j, k)$. The Koecher principle also applies to these modular forms: sections of $\mathbb{E}_{2}^{\rho}$ over $\mathcal{A}_{2}$ extend over $\tilde{\mathcal{A}}_{2}$, see [7, Prop. 1.5, p. 140]. We write

$$
M_{j, k}\left(\Gamma_{2}\right)=H^{0}\left(\tilde{\mathcal{A}}_{2} \otimes \mathbb{F}_{3}, \operatorname{Sym}^{j}\left(\mathbb{E}_{2}\right) \otimes \operatorname{det}\left(\mathbb{E}_{2}\right)^{k}\right)
$$

and we consider the $\mathcal{R}_{2}\left(\mathbb{F}_{3}\right)$-module

$$
M=\oplus_{j, k} M_{j, k}\left(\Gamma_{2}\right) .
$$

It is even a ring. The map (2.3) can be extended to a map from $M$ to the ring of covariants. Here a covariant can be described as an invariant for the action of $\operatorname{GL}(V)$ on $V \oplus \operatorname{Sym}^{6}(V)$. Alternatively, covariants can be obtained by taking an equivariant embedding of an irreducible $\mathrm{GL}(V)$-representation $U \rightarrow \operatorname{Sym}^{d}\left(\operatorname{Sym}^{6}(V)\right)$, or equivalently, an equivariant map

$$
\varphi: \mathbb{F}_{3} \rightarrow \operatorname{Sym}^{d}\left(\operatorname{Sym}^{6}(V)\right) \otimes U^{\vee}
$$

and then $\Phi=\varphi(1)$ is a covariant. If $U$ is an irreducible representation of highest weight $\left(w_{1}, w_{2}\right)$ then one may view $\Phi$ as a homogeneous form in $a_{0}, \ldots, a_{6}$ of degree $d$ and in $x_{1}, x_{2}$ of degree $w_{1}-w_{2}$, see [2,9,19]. For example, taking $U=\operatorname{Sym}^{6}(V)$ and $d=1$ yields the covariant $\Phi=f$, the universal binary sextic. Covariants form a ring $\mathcal{C}$ that was much studied in the 19th and early 20th century. Grace and Young determined generators of this ring in [9].

The maps $\mathcal{R}_{2}\left(\mathbb{F}_{3}\right) \rightarrow I \rightarrow \mathcal{R}_{2}\left(\mathbb{F}_{3}\right)_{\chi_{10}}$ now extend to

$$
M \stackrel{\mu}{\longrightarrow} \mathcal{C} \stackrel{v}{\longrightarrow} M_{\chi_{10}}
$$

where $M_{\chi_{10}}$ is obtained from $M$ by admitting powers of $\chi_{10}$ as denominators. We have $v \circ \mu=\operatorname{id}_{M}$.

The image under $v$ of the covariant $f$, the universal binary sextic, is a rational modular form $\chi_{6,-2}$, that is, a rational section of $\operatorname{Sym}^{6}\left(\mathbb{E}_{2}\right) \otimes \operatorname{det}\left(\mathbb{E}_{2}\right)^{-2}$ that is regular after multiplication by an appropriate power of $\chi_{10}$. The power -2 comes from the twisting used in the description of the stack quotient $\left[\mathcal{X}^{0} / \mathrm{GL}(V)\right]$, where $\mathcal{X}^{0} \subset \operatorname{Sym}^{6}(V) \otimes \operatorname{det}(V)^{-2}$, see [3, Section 3, p. $3]$. 
This construction was given in [2] in characteristic zero and yields a meromorphic modular form, here denoted $\varphi_{6,-2}$, that becomes holomorphic after multiplication by $\chi_{10}$. The reduction of the characteristic zero rational modular form $\varphi_{6,-2}$ yields a rational modular form in characteristic 3 . This implies that $\chi_{6,-2}$ becomes regular after multiplication by $\chi_{10}$. We can write the form $\chi_{6,-2}$ locally on $\mathcal{A}_{2} \otimes \mathbb{F}_{3}$ symbolically as

$$
\chi_{6,-2}=\sum_{i=0}^{6} \alpha_{i} X_{1}^{6-i} X_{2}^{i},
$$

where the monomials $X_{1}^{6-i} X_{2}^{i}$ are dummies to indicate the coordinates in the fibres of $\operatorname{Sym}^{6}\left(\mathbb{E}_{2}\right) \otimes \operatorname{det}\left(\mathbb{E}_{2}\right)^{-2}$. Here we view $\alpha_{i}$ locally as a rational function on $\mathcal{A}_{2} \otimes \mathbb{F}_{3}$. Using the local expression (2.4) one can give the image $v(T)$ of an invariant $T=T\left(a_{0}, \ldots, a_{6}\right)$ locally by $T\left(\alpha_{0}, \ldots, \alpha_{6}\right)$.

We note that interchanging $X_{1}$ and $X_{2}$ induces an involution replacing $\alpha_{i}$ by $\alpha_{6-i}$.

Comparing with the characteristic 0 case and using semi-continuity we see that the orders of the rational functions $\alpha_{i}$ along the divisor $H_{1}$ are at least equal to the orders of their complex analogues along $H_{1}$. The Fourier expansion in characteristic 0 given in [2, page 1658] implies the following inequalities for the orders of $\alpha_{i}$ along $H_{1}$ in characteristic 3:

$$
\operatorname{ord}_{H_{1}}\left(\alpha_{0}, \ldots, \alpha_{6}\right)=(\geq 2, \geq 1, \geq 0, \geq-1, \geq 0, \geq 1, \geq 2) .
$$

Moreover, the symmetry that interchanges $x_{1}$ and $x_{2}$ implies that the orders of $\alpha_{i}$ and $\alpha_{6-i}$ along $H_{1}$ are equal. Another way to see the estimates for the orders is by developing $\chi_{6,8}=$ $\chi_{6,-2} \chi_{10}$ along the locus $\mathcal{A}_{1,1} \otimes \mathbb{F}_{3} \subset \mathcal{A}_{2} \otimes \mathbb{F}_{3}$. Since the pullback of the Hodge bundle $\mathbb{E}_{2}$ to $\mathcal{A}_{1} \times \mathcal{A}_{1}$ via $\mathcal{A}_{1}^{2} \rightarrow \mathcal{A}_{1,1} \subset \mathcal{A}_{2}$ is $\oplus_{i=0}^{6} p_{1}^{*}\left(\mathbb{E}_{1}\right)^{i} \otimes p_{2}^{*}\left(\mathbb{E}_{1}\right)^{6-i}$ the restriction of $\alpha_{i} \chi_{10}$ lies in $S_{14-i}\left(\Gamma_{1}\right) \otimes S_{8+i}\left(\Gamma_{1}\right)$ and this is zero. The next Taylor term in the Taylor development along $\mathcal{A}_{1,1}$ lies in $S_{15-i}\left(\Gamma_{1}\right) \otimes S_{9+i}\left(\Gamma_{1}\right)$ and this is zero for $i \neq 3$.

The ring of invariants $I$ for the action of GL $(V)$ on $\operatorname{Sym}^{6}(V)$ in characteristic 3 is generated by invariants $A, B, C, D$ and $E$ of degree 2, 4, 6, 10 and 15, see e.g. [11] or [8]. The invariants $A, B, C, D$ that we use here can be expressed in the reductions modulo 3 of the invariants $J_{2}, J_{4}, J_{6}$ et $J_{10}$ given in [15]: $A=-J_{2}(\bmod 3), B=-J_{4}(\bmod 3), C=$ $-J_{6}-A^{3}(\bmod 3), D=J_{10}(\bmod 3)$. The invariant $E$ can be found in [12, p. 848].

The invariant $A$ has the form $A=a_{1} a_{5}-a_{2} a_{4}$. We know of the existence of a modular form $\psi_{2}$ of weight 2 . Under the map $\mu$ it must map to a non-zero multiple of $A$. We fix $\psi_{2}$ by requiring $\mu\left(\psi_{2}\right)=A$. The restriction to $H_{1}$ of the Hasse invariant $\psi_{2}$ is a non-zero multiple of $\operatorname{Sym}^{2}\left(b_{2}\right)$, with $b_{2}$ the Hasse invariant for $g=1$, hence $\psi_{2}$ does not vanish identically on $H_{1}$. and

By the inequalities (2.5) and the expression for $A$ we see that $\operatorname{ord}_{H_{1}}\left(\alpha_{2}\right)=0=\operatorname{ord}_{H_{1}}\left(\alpha_{4}\right)$

$$
\operatorname{ord}_{H_{1}}\left(\alpha_{0}, \ldots, \alpha_{6}\right)=(\geq 2, \geq 1,0, \geq-1,0, \geq 1, \geq 2) .
$$

In degree 4 we find another invariant $B$, not a multiple of $A^{2}$ :

$$
\begin{aligned}
B= & 2 a_{0} a_{1} a_{5} a_{6}+a_{0} a_{2} a_{4} a_{6}+2 a_{0} a_{2} a_{5}^{2}+2 a_{0} a_{4}^{3}+2 a_{1}^{2} a_{4} a_{6}+2 a_{1} a_{2} a_{4} a_{5} \\
& +a_{1} a_{3}^{2} a_{5}+a_{1} a_{3} a_{4}^{2}+2 a_{2}^{3} a_{6}+a_{2}^{2} a_{3} a_{5}+a_{2}^{2} a_{4}^{2}+2 a_{2} a_{3}^{2} a_{4} .
\end{aligned}
$$

Since we know $\operatorname{dim} M_{4}\left(\Gamma_{2}\right)=1$ there cannot be a regular modular form in weight 4 that is not a multiple of $\psi_{2}^{2}$. This implies that $\operatorname{ord}_{H_{1}}\left(\alpha_{3}\right)<0$ and hence ord $H_{1}\left(\alpha_{3}\right)=-1$. Thus $B=\left(a_{1} a_{5}-a_{2} a_{4}\right) a_{3}^{2}+\left(a_{1} a_{4}^{2}+a_{2}^{2} a_{5}\right) a_{3}+\cdots$ defines a rational modular form $\chi_{B}=v(B)$ 
of weight 4 with order -2 along $H_{1}$. Since $\chi_{10}$ vanishes with multiplicity 2 along $H_{1}$ we thus find that

$$
\chi_{14}:=\chi_{B} \chi_{10}
$$

is a regular modular form of weight 14 .

The vector space of invariants of degree 6 is generated by $A^{3}, A B$ and an invariant $C$

$$
C=2 a_{3}^{6}+A a_{3}^{4}+2\left(a_{1} a_{4}^{2}+a_{2}^{2} a_{5}\right) a_{3}^{3}+\cdots
$$

and we see that $\chi_{C}=v(C)$ has order -6 along $H_{1}$. In degree 10 there is a new invariant

$$
D=\left(a_{1} a_{5}\right)^{3} a_{3}^{4}+\left(a_{0} a_{2}^{3} a_{5}^{3}+a_{1}^{3} a_{4}^{3} a_{6}+2 a_{1}^{3} a_{4}^{2} a_{5}^{2}+2 a_{1}^{2} a_{2}^{2} a_{5}^{3}\right) a_{3}^{3}+\cdots
$$

yielding a modular form that vanishes with multiplicity $\geq 2$ on $H_{1}$. Indeed, since $\alpha_{1} \alpha_{5}$ vanishes with multiplicity $\geq 2$ the first term $\left(\alpha_{1} \alpha_{5}\right)^{3} \alpha_{3}^{4}$ vanishes with order $\geq 2$; the next terms also vanish with order $\geq 2$ as one easily checks. Therefore $\chi_{D}$ is regular and vanishes with multiplicity $\geq 2$. Since $\chi_{D}$ is not zero, it must be a multiple of $\chi_{10}$ and then vanishes on $H_{1}$ with multiplicity 2 . This implies that the order of vanishing of $\alpha_{1}$ and $\alpha_{5}$ along $H_{1}$ is 1 .

Corollary 1 We have $\operatorname{ord}_{H_{1}}\left(\alpha_{0}, \ldots, \alpha_{6}\right)=(\geq 2,1,0,-1,0,1, \geq 2)$.

We fix $\chi_{10}$ by setting it equal to $\chi_{D}=v(D)$. This fixes $\chi_{14}$ too.

In a similar manner one checks that the rational modular form $\psi_{S}=v(S)$ with $S$ equal to

$$
S=B^{3}+A^{3} C-A^{2} B^{2}=\left(a_{1} a_{4}^{2}+a_{2}^{2} a_{5}\right)^{3} a_{3}^{3}+\cdots
$$

is regular too. We put $\psi_{12}=\psi_{S}$. We thus find a 3-dimensional subspace of $M_{12}\left(\Gamma_{2}\right)$ generated by $\psi_{2}^{6}, \psi_{2} \chi_{10}$ and $\psi_{12}$. From the fact that $B$ and $D$ are not divisible by $A$ we see that $\chi_{14}$ does not lie in $\psi_{2} M_{12}\left(\Gamma_{2}\right)$. Therefore $\operatorname{dim} M_{12}\left(\Gamma_{2}\right)<\operatorname{dim} M_{14}\left(\Gamma_{2}\right)$. Since we know by (2.1) that $\operatorname{dim} M_{14}\left(\Gamma_{2}\right) \leq 4$ we conclude that $\operatorname{dim} M_{12}\left(\Gamma_{2}\right)=3$.

A further generator is

$$
\chi_{36}=v\left(C D^{3}\right)=\chi_{C} \chi_{10}^{3} .
$$

Since the orders of $\chi_{C}$ and $\chi_{10}$ along $H_{1}$ are -6 and 2 the modular form $\chi_{36}$ is regular and does not vanish identically on $H_{1}$. The modular form $\chi_{36}$ is not contained in the subring generated by $\psi_{2}, \chi_{10}, \psi_{12}$ and $\chi_{14}$ as one sees by looking at the invariants. We have the identity

$$
\left(B^{3}+A^{3} C-A^{2} B^{2}\right) D^{3}=B^{3} D^{3}+A^{3} C D^{3}-A^{2} D B^{2} D^{2}
$$

by which we can express $\psi_{12} \chi_{10}^{3}$ in the other generators:

$$
\psi_{12} \chi_{10}^{3}=\chi_{14}^{3}+\psi_{2}^{3} \chi_{36}-\psi_{2}^{2} \chi_{10} \chi_{14}^{2} .
$$

Since $A, B, C, D$ are generators of the ring of invariants and are algebraically independent the forms $\psi_{2}, \chi_{10}, \psi_{12}, \chi_{14}$ are algebraically independent. The form $\chi_{36}$ then satisfies the algebraic relation (2.6) and since there is no non-trivial relation of lower weight involving $\chi_{36}$ it implies that this relation generates the ideal of relations between the generators $\psi_{2}$, $\chi_{10}, \psi_{12}, \chi_{14}$ and $\chi_{36}$.

The forms $\psi_{2}, \chi_{10}, \psi_{12}, \chi_{14}$ and $\chi_{36}$ generate a subring $R^{\mathrm{ev}}$ of the ring $\mathcal{R}_{2}^{\mathrm{ev}}\left(\mathbb{F}_{3}\right)$ with generating function

$$
G=\frac{\left(1-t^{42}\right)}{\left(1-t^{2}\right)\left(1-t^{10}\right)\left(1-t^{12}\right)\left(1-t^{14}\right)\left(1-t^{36}\right)} .
$$


and by the Riemann-Roch theorem we have $\operatorname{dim} M_{k}\left(\Gamma_{2}\right)=k^{3} / 1080+O\left(k^{2}\right)$ for even $k$. Note that

$$
\frac{42}{2 \cdot 10 \cdot 12 \cdot 14 \cdot 36}=\frac{1}{2880} .
$$

On the other hand we have $c_{1}(L)^{3}=1 / 2880$, see [22, p. 74]. We can use the degree of $\operatorname{Proj}\left(\mathcal{R}_{2}^{\mathrm{ev}}\left(\mathbb{F}_{3}\right)\right)$ to show that there cannot be more generators of $\mathcal{R}_{2}^{\mathrm{ev}}\left(\mathbb{F}_{3}\right)$, but one can see this also in a more elementary way as follows.

Let $d(k)=\operatorname{dim} M_{k}\left(\Gamma_{2}\right)$ and $r(k)=\operatorname{dim} R_{k}$ where $R_{k}=R^{\mathrm{ev}} \cap M_{k}\left(\Gamma_{2}\right)$.

Proposition 1 We have $d(k)=r(k)$ for even $k \geq 0$.

Proof We know that $d(k) \geq r(k)$ for even $k$ and $d(k)=r(k)$ for even $0 \leq k \leq 14$. Suppose by induction that $d(k)=r(k)$ for even $k \leq m$. The exact sequence (2.1) gives the upper bound $d(k) \leq r(k-10)+c(k)(c(k)+1) / 2$ for $k \leq m+10$, where $c(k)=\operatorname{dim} M_{k}\left(\Gamma_{1}\right)=\lfloor k / 12\rfloor+1$. Using the generating function $G$ one sees that $r(k)-r(k-10)=c(k)(c(k)+1) / 2$ for $k \neq \equiv 0(\bmod 12)$ and $k \neq \equiv 2(\bmod 12)$. Hence $d(k)=r(k)$ for even $k \leq m+10$ with $k \not \equiv 0,2(\bmod 12)$. But we have

$$
d(k+2)-d(k) \geq r(k+2)-r(k),
$$

as we show in the next lemma. This proves $d(k)=r(k)$ for even $k \leq m+10$. Therefore we conclude the proof by induction.

Lemma 1 We have $d(k+2)-d(k) \geq r(k+2)-r(k)$ for even $k \geq 0$.

Proof We can write $R_{k+2}=\psi_{2} R_{k} \oplus N_{k+2}$ with $N_{k+2}$ the subspace with basis the forms $\chi_{10}^{a} \psi_{12}^{b} \chi_{14}^{c} \chi_{36}^{d}$ with $a, b, c, d \geq 0$ and $c \leq 2$ in view of the relation (2.6). Then we have $\operatorname{dim} N_{k+2}=r(k+2)-r(k)$. The inequality $d(k+2)-d(k) \geq \operatorname{dim} N_{k+2}$ follows from the fact that $N_{k+2} \cap \psi_{2} M_{k}\left(\Gamma_{2}\right)=(0)$. To see this fact, suppose that $f \in M_{k}\left(\Gamma_{2}\right)$ such that $f \notin R_{k}$ and $\psi_{2} f \in R_{k+2}$. Then $\psi_{2} f=P$ with $P$ a sum of monomials $\chi_{10}^{a} \psi_{12}^{b} \chi_{14}^{c} \chi_{36}^{d}$ with $c \leq 2$. Then $P=v(Q)$ with $Q$ a polynomial in

$$
D, B^{3}+A^{3} C-A^{2} B^{2}, B D, C D^{3} \text {. }
$$

Since $P=\psi_{2} f$ this polynomial must be divisible by $A$. But this implies that if $Q \neq 0$ then it must have at least one monomial with $c \geq 3$, but we excluded this.

The invariant $E$ of degree 15 is of the form

$$
E=\left(a_{1} a_{4}^{2}-a_{2}^{2} a_{5}\right)^{3} a_{3}^{6}+\cdots
$$

and $v(E)$ has order -3 along $H_{1}$. Therefore

$$
\chi_{35}:=v\left(E D^{2}\right)
$$

is a regular modular form. It vanishes on $H_{1}$ and on the Humbert surface $H_{4}$ of discriminant 4, both with multiplicity 1 . The surfaces $H_{1}$ and $H_{4}$ parametrize abelian surfaces that possess an extra involution. Locally near $H_{4}$ the extra automorphism corresponds to the symmetry that interchanges $x_{1}$ and $x_{2}$.

We know that the cycle class of $2 H_{4}$ on $\mathcal{A}_{2}^{*} \otimes \mathbb{F}_{3}$ is $60 \lambda_{1}$, see [21, Prop. 3.3, p. 217]. Therefore the divisor of $\chi_{35}$ is $H_{1}+H_{4}$ and since the closure of $H_{1}$ contains the 1-dimensional cusp $\chi_{35}$ is a cusp form. Then $\chi_{35}^{2}$ is of even weight, hence can be expressed as a polynomial 
in $\psi_{2}, \chi_{10}, \psi_{12}, \chi_{14}$ and $\chi_{36}$. If $\psi$ is an odd weight modular form then it must vanish on $H_{1}$ and $H_{4}$, hence it will be divisible by $\chi_{35}$.

The relation between the space of binary sextics and the moduli space $\overline{\mathcal{M}}_{2}$ (see for example [3, Section 4]) implies that a modular form $\chi$ is a cusp form if and only if the invariant $\mu(\chi)$ is divisible by the discriminant $D$ in $I$. From the form of the generators one easily sees that $\chi_{10}, \chi_{14}, \chi_{36}$ and $\chi_{35}$ generate the ideal of cusp forms. This completes the proof.

Remark 1 One can use the knowledge of the dimensions of $M_{k}\left(\Gamma_{2}\right)$ to deduce non-vanishing of $H^{1}\left(\tilde{\mathcal{A}}_{2} \otimes \mathbb{F}_{3}, L^{k}\right)$ for certain values of $k$. The short exact sequence of sheaves on $\tilde{\mathcal{A}}_{2} \otimes \mathbb{F}_{3}$

$$
0 \rightarrow L^{k} \otimes \mathcal{O}\left(-\bar{V}_{1}\right) \rightarrow L^{k} \rightarrow L_{\mid \bar{V}_{1}}^{k} \rightarrow 0
$$

gives rise to a long exact sequence which can be identified with

$$
0 \rightarrow M_{k-2}\left(\Gamma_{2}\right) \rightarrow M_{k}\left(\Gamma_{2}\right) \rightarrow H^{0}\left(\bar{V}_{1}, L^{k}\right) \rightarrow H^{1}\left(\tilde{\mathcal{A}}_{2} \otimes \mathbb{F}_{3}, L^{k-2}\right) \rightarrow \cdots
$$

For example, if $\operatorname{dim} M_{k-2}\left(\Gamma_{2}\right)=\operatorname{dim} M_{k}\left(\Gamma_{2}\right)$ we get an injection $H^{0}\left(\bar{V}_{1}, L^{k}\right) \rightarrow H^{1}\left(\tilde{\mathcal{A}}_{2} \otimes\right.$ $\left.\mathbb{F}_{3}, L^{k-2}\right)$ and if $k \equiv 0(\bmod 4)$ and $k \geq 0$ one can show that $H^{0}\left(\bar{V}_{1}, L^{k}\right) \neq 0$ by showing that $H^{0}\left(\bar{V}_{1}[2], L^{k}\right)^{\mathfrak{S}_{6}} \neq(0)$, the space of invariants under the symmetric group $\mathfrak{S}_{6}$ acting on $H^{0}\left(\bar{V}_{1}[2], L^{k}\right)$ with $V_{1}[2]$ the 3 -rank $\leq 1$ locus in the level 2 moduli space $\tilde{\mathcal{A}}_{2}$ [2]. Thus for example, $H^{1}\left(\tilde{\mathcal{A}}_{2} \otimes \mathbb{F}_{3}, L^{14}\right) \neq(0)$.

Acknowledgements The author thanks Fabien Cléry for helpful correspondence. He thanks YMSC at Tsinghua University where part of this work was done for hospitality. Finally thanks are due to the referee for his/her remarks.

Open Access This article is licensed under a Creative Commons Attribution 4.0 International License, which permits use, sharing, adaptation, distribution and reproduction in any medium or format, as long as you give appropriate credit to the original author(s) and the source, provide a link to the Creative Commons licence, and indicate if changes were made. The images or other third party material in this article are included in the article's Creative Commons licence, unless indicated otherwise in a credit line to the material. If material is not included in the article's Creative Commons licence and your intended use is not permitted by statutory regulation or exceeds the permitted use, you will need to obtain permission directly from the copyright holder. To view a copy of this licence, visit http://creativecommons.org/licenses/by/4.0/.

\section{References}

1. Böcherer, S., Nagaoka, S.: On mod $p$ properties of Siegel modular forms. Math. Ann. 338, 421-433 (2007)

2. Cléry, F., Faber, C., van der Geer, G.: Covariants of binary sextics and vector-valued Siegel modular forms of genus two. Math. Ann. 369, 1649-1669 (2017). arXiv:1606.07014

3. Cléry, F., Faber, C., van der Geer, G.: Covariants of binary sextics and modular forms of degree 2 with character. Math. Comput. 88(319), 2423-2441 (2019). arXiv:1803.05624

4. Cléry, F., van der Geer, G.: Modular forms of degree two and curves of genus two in characteristic two (2020). arXiv:2003.00249 (to appear in IMRN)

5. Deligne, P.: Courbes elliptiques: Formulaire (d'après J. Tate). In: Modular functions IV. Lecture notes in mathematics, vol. 476, pp. 53-73. Springer, Berlin (1975)

6. Ekedahl, T., van der Geer, G.: Cycle classes of the E-O stratification on the moduli of abelian varieties. In: Tschinkel, Y., Zarhin, Y. (eds.) Algebra, arithmetic, and geometry. Progress in Mathematics, vol. 269. Birkhäuser, Basel (2010)

7. Faltings, G., Chai, C.L.: Degeneration of abelian varieties. Ergebnisse der Math., vol. 22. Springer, New York (1990)

8. Geyer, W.: Invarianten binärer Formen. In: Classification of algebraic varieties and compact complex manifolds. Lecture Notes in Math., vol. 412, pp. 36-69. Springer, Berlin (1974)

9. Grace, J.H., Young, A.: The algebra of invariants. Cambridge University Press, Cambridge (1903)

10. Ichikawa, T.: Siegel modular forms of degree 2 over rings. J. Number Theory 129, 818-823 (2009) 
11. Igusa, J.-I.: Arithmetic variety of moduli for genus two. Ann. Math. 72, 612-649 (1960)

12. Igusa, J.-I.: Modular forms and projective invariants. Am. J. Math. 89, 817-855 (1967)

13. Igusa, J.-I.: On the ring of modular forms of degree two over. Am. J. Math. 101, 149-183 (1979)

14. Lercier, R., Ritzenthaler, C.: Siegel modular forms of degree three and invariants of ternary quartics (2020). arXiv:1907.07431

15. Liu, Q.: Modèles minimaux des courbes de genre deux. J. Reine Angew. Math. 453, 137-164 (1994)

16. Mumford, D.: Towards an enumerative geometry of the moduli space of curves. In: Arithmetic and geometry, Papers dedicated to I. R. Shafarevich, vol. II. Geometry. Progress in Math., vol. 36, pp. 271328. Birkhäuser, Boston (1983)

17. Nagaoka, S.: Note on mod $p$ Siegel modular forms. I, II. Math. Zeitschrift 235, 405-420 (2000)

18. Nagaoka, S.: Note on mod $p$ Siegel modular forms. I, II. Math. Zeitschrift 251, 821-826 (2005)

19. Springer, T.A.: Invariant theory. Lecture notes in mathematics, vol. 585. Springer, New York (1977)

20. Tsuyumine, S.: On Siegel modular forms of degree three. Am. J. Math. 108, 755-862 (1986) [Addendum to "On Siegel modular forms of degree three." Amer. J. Math. 108 (1986), pp. 1001-1003]

21. van der Geer, G.: Hilbert modular surfaces Ergebnisse der Math., vol. 16. Springer, Berlin (1988)

22. van der Geer, G.: Cycles on the moduli space of abelian varieties. In: Moduli of curves and abelian varieties. Aspects Math., vol. E33. Vieweg, Braunschweig (1999)

Publisher's Note Springer Nature remains neutral with regard to jurisdictional claims in published maps and institutional affiliations. 